\title{
PROPUESTA DE SISTEMATIZACIÓN AGROHIDROLÓGICA EN CUENCAS DE LLANURA CON ÉNFASIS PREDIAL Y CONSORCIADO
}

\author{
Damiano, F. ${ }^{1} ;$ Parodi, G. N. ${ }^{2}$; Taboada, M. A. ${ }^{3,4}$ \& Prieto Garra, D. ${ }^{5}$
}

\begin{abstract}
RESUMEN
Existe escasa tecnología para el manejo de aguas superficiales en áreas de planicie extrema e inundable, aumentada por la controversia de opinión en cuanto al modo de encarar la solución de los excesos y déficit hídricos. La Agrohidrología modular considera el importante rol que cumple el agua en el sistema de producción, manejándola mediante un conjunto de prácticas conservacionistas rurales, de ingeniería agronómica y civil destinadas a controlar los anegamientos periódicos en campos bajos o en llanuras extensas. La unidad agrohidrológica básica es el diseño de "circuitos hidrológicos" que evita la transferencia anárquica de agua a otras unidades, circuitos o sistemas hídricos, mediante un conjunto de obras estructurales de encauzamiento, conducción, retención, expansión y evacuación. Estableciendo un marco hídrico y edafológico apropiado para acumular los excedentes de agua en sectores menos productivos, protegiendo ambientes con riesgo de hidromorfismo y halomorfismo y su reincorporación a los esquemas agronómicos productivos.
\end{abstract}

Palabras clave: región pampa inundable, sistema hidrológico no típico, circuitos hidrológicos, estructuras de tierra.

\footnotetext{
ABSTRACT

Proposal for agro-hydrological systematization in basins plaines with emphasis predial and consortial.

Available and technology for surface water management in such large plains is still scarce or site-specific. Controversy often arises about how to find the best solution to manage alternated periods with water excesses and droughts in the same area. The best technical solution is "agrohydrology", which can be considered a "conservation / interventionist" approach attempting to

1. Instituto de Clima y Agua, CIRN, INTA

2. Faculty of Geo-Information Science and Earth Observation of the University of Twente, Enschede, Países Bajos.

3. Instituto de Suelos, CIRN, INTA.

4. CONICET

5. Estación Experimental Agropecuaria, INTA, Santiago del Estero.

Manuscrito recibido el 20 de octubre de 2016 y aceptado para su publicación el 7 de febrero de 2017.
} 
manage the soil-plant-atmosphere system. The concept here proposed is water management in lowlands using "hydrologic circuits" capable of temporarily storing great water volumes to retard downstream evacuation. This requires the design and engineering calculation of different earth structures for captation, conduction and temporal storage of excess water, using combined "engineering-rural" management. This solution attempts to cope the typical climatic alternation of the flooding Pampa region: floods and droughts occurring in subsequent seasons, and both related to groundwater and soil salinization processes in this region. Water management by agrohydrology can be then regarded as a first step before the implementation of classical soil reclamation techniques in large plains.

Key words: flooding Pampa region, atypical hydrological system, hydrologic circuits, earth structures.

\section{Cuenca hidrológica: planificación territorial y manejo del agua}

\section{¿Qué es una cuenca superficial?}

El término cuenca superficial imbrífera, cuenca de drenaje o simplemente cuenca son distintas denominaciones de la porción de territorio que drena hacia un punto (salida o exutorio). La cuenca actúa análogamente a un embudo en el que el agua que ingresa en forma de precipitación escurre superficialmente a un único punto de salida. El límite de una cuenca superficial, llamado divisoria de aguas, está determinado por el relieve del área que la separa topográficamente de las otras cuencas adyacentes.

La cuenca hidrológica incorpora las aguas subterráneas y se define como la unidad territorial donde las aguas superficiales y subterráneas forman parte de un ciclo hidrológico común donde la diferencia entre los caudales de aguas entrantes y salientes en un tiempo establecido es igual a la variación del volumen de agua en esa unidad. Esta relación queda expresada a través de un balance hídrico.

\section{¿Qué es manejo de cuencas?}

La filosofía del manejo de cuencas (watershed management) es establecer estructuras y estrategias destinadas a regular la descarga de agua de una cuenca hidrológica. El objetivo es alterar algunas o todas las variables que componen el balance hídrico antes mencionado. En áreas con excesos de agua se busca incrementar la retención de agua en los suelos, análogamente a una esponja, y así disminuir la salida de agua en cantidad, incrementando el tiempo de permanencia en la cuenca, que trae asociado una variación en la calidad del agua y un aumento de la evaporación. Se reduce de esta manera el escurrimiento rápido, causante de inundaciones, en favor de un aumento del flujo subsuperficial. Por el contrario en áreas con déficit de agua, se busca impedir la infiltración del agua para poder captarla y retenerla en algún receptáculo. En todos los casos estos objetivos se orientan a la utilización de la cuenca como almacenadora (catchment area) de agua para diferentes fines, como consumo humano (cuencas municipales), rural e industrial y para reducir el impacto de las crecidas protegiendo así zonas vulnerables 